\title{
Tracheal Metastasis from Rectal Cancer: A Case Report and Review of the Literature
}

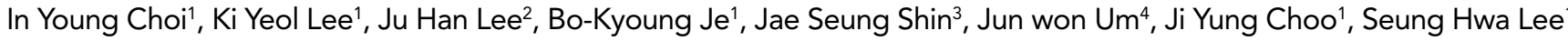 \\ ${ }^{1}$ Department of Radiology, Ansan Hospital, Faculty of Medicine, Korea University, Republic of Korea \\ ${ }^{2}$ Department of Pathology, Ansan Hospital, Faculty of Medicine, Korea University, Republic of Korea \\ ${ }^{3}$ Department of Thoracic and Cardiovascular Surgery, Ansan Hospital, Faculty of Medicine, Korea University, Republic of Korea \\ ${ }^{4}$ Department of General Surgery, Ansan Hospital, Faculty of Medicine, Korea University, Republic of Korea
}

\section{ABSTRACT}

The trachea is an uncommon site of metastasis from colorectal carcinoma. A few cases have been reported in the literature, but these focused mostly on the clinical aspects without detailing radiologic and histologic findings. The authors describe a 70-year-old woman who was diagnosed with trachea metastasis from a primary rectal cancer. We present the contrast-enhanced chest computed temography (CT), including volume-rendered image, as well as bronchoscopic findings.

Key Word: Tracheal tumor, metastasis, rectal cancer

\section{Introduction}

Metastases from nonpulmonary solid tumors to the trachea are uncommon (1). There have been reported cases of metastases from breast cancer, renal cell carcinoma, melanoma, and colorectal carcinoma (2-5). Advances in postoperative chemotherapy have increased the survival rate in patients with colorectal carcinoma and have revealed a new population with endobronchial metastases which may arise in the later stages of the disease (6). Nevertheless, endotracheal manifestation is so rare that the incidence cannot be established. To our knowledge, three cases have been reported in the English literature, and these reports shared only clinical details without detailed radiologic and histologic findings (2, 7). Therefore, we present the 70-year-old woman with endotracheal metastasis from rectal cancer.

\section{Case Report}

A 70-year-old woman was diagnosed with stage T3N1Mo rectal cancer and underwent abdomino-perineal resection followed by concurrent chemoradiotherapy 11 years previously. She had been asymptomatic until 9 years after the diagnosis when a single pulmonary nodule was identified in the right upper lobe of the lung without mediastinal lymphadenopathy. A subsequent chest CT scan revealed enlargement of the nodule after a year, and wedge resection was performed and it was confirmed that the mass was a pulmonary metastasis from adenocarcinoma of the rectum. Two years later, the patient was referred to the pulmonology depart- ment with blood-tinged sputum and dyspnea. Chest CT scan (Brilliance $^{\mathrm{TM}}$ 64, Philips Medical System, Cleveland, OH, USA) taken for further evaluation of blood tinged sputum showed a $1.3 \mathrm{~cm} \times 0.8 \mathrm{~cm}$ intraluminal lesion arising from the right lateral wall of the upper trachea with homogeneous soft tissue attenuation on a pre-enhanced scan and homogeneous enhancement by $50 \mathrm{HU}$ with contrast (Figure 1A). A new 2 $\mathrm{cm}$-sized pulmonary mass was also noted in the right upper lobe at the previous resection site. The volume-rendered image depicting an ovoid intraluminal mass arising from the lateral tracheal wall (Figure 1B) was well-correlated with bronchoscopic findings that showed a polypoid tracheal mass and resultant luminal narrowing by approximately two-thirds the diameter of the upper trachea (Figure 1C). The clinician performed local excision with a laser microscope rather than the usual bronchoscopic biopsy to prevent bleeding from the mass. Lobectomy of the right upper lobe for the pulmonary mass was performed at the same time. Histological examination (Hematoxylin \& Eosin staining) of the tracheal mass showed well-formed glands (white arrows) with glandular fusion (hematoxylin-eosin, original $\times 200$ ). The tumor cells are positive for CK 20 but negative for CK7 and the investigation revealed metastatic adenocarcinoma with the same histology as those of the primary rectal cancer and previous pulmonary metastasis (Figure 1D). The tracheal lesion could not be excised completely, so the patient received palliative radiotherapy. The patient has lived for 11 months since the palliative radiotherapy without any evidence of local tumor recurrence and distant metastases. 

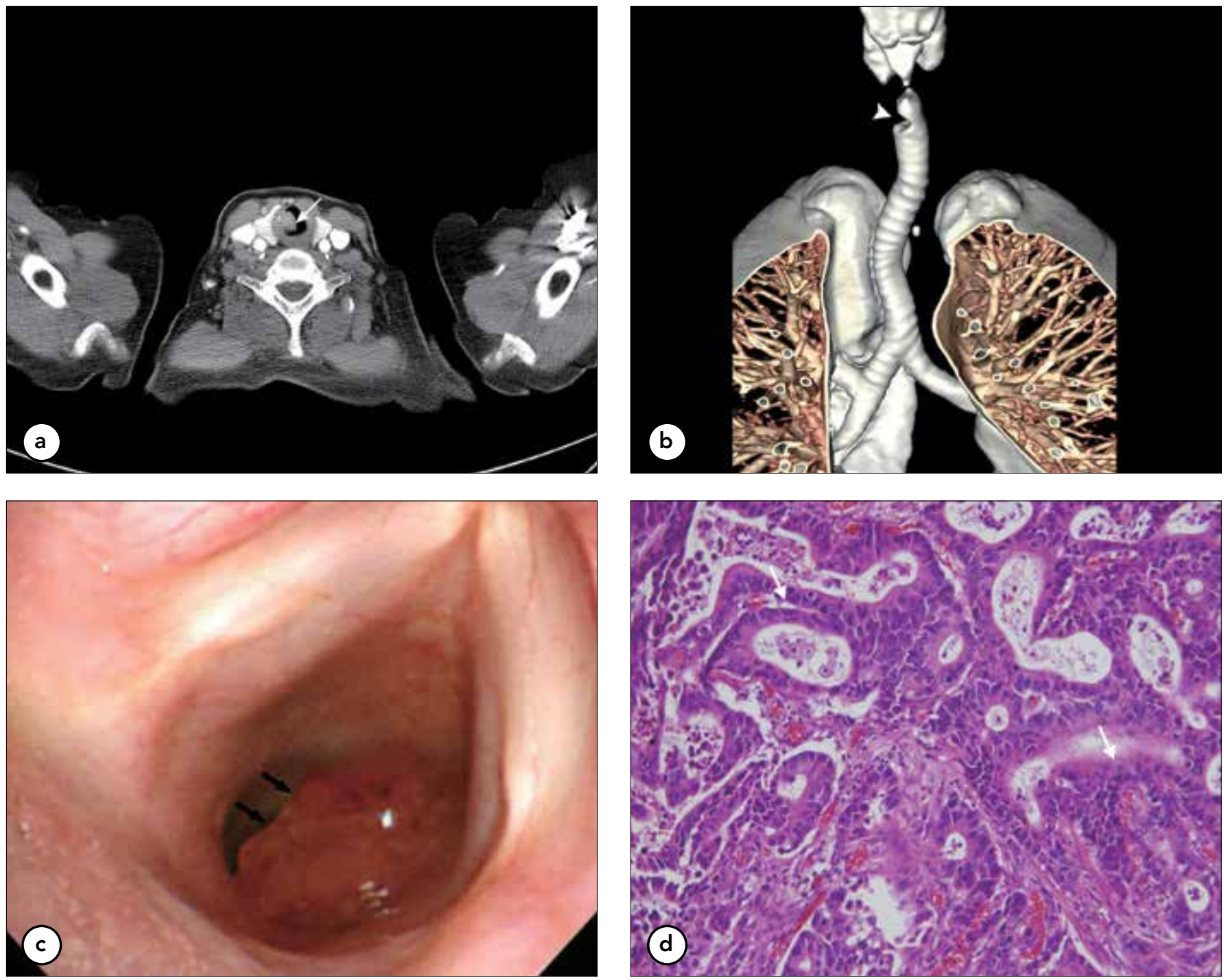

Figure 1. 70-year-old woman with an endotracheal metastasis from rectal cancer a) Contrast enhanced axial CT scan showed a $1.3 \mathrm{~cm} \times 0.8 \mathrm{~cm}$ intraluminal mass (arrow) arising from the right lateral tracheal wall with homogeneous enhancement, about 50HU comparing precontrast enhanced CT image. b) The volume-rendered image depicted an ovoid intraluminal mass (arrowhead) arising from lateral tracheal wall in the upper level of the trachea. c) Bronchoscopy revealed a polypoid lesion. (black arrows) in the upper trachea with occlusion of approximately two-thirds the diameter of the endotracheal lumen. d) Tumor histology shows well-formed glands (white arrows) with glandular fusion (hematoxylin-eosin, original $\mathbf{x} 200$ ). There are foamy histiocytes and necrotic material in the intraluminal area. These findings are consistent with previous colon cancer morphology.

\section{Discussion}

Pulmonary metastases of colorectal cancer have been reported in $15 \%$ of patients; however endotracheal metastases are uncommon (7). According to Chong et al. (8) the overall incidence of tracheal metastasis was $0.44 \%$ in surgically-resected non-small cell lung cancer. The prevalence of metastasis from nonpulmonary malignancies to the tracheobronchial tree is only $2 \%$ at autopsy (7). Although the reason is unclear, Fournel et al. (6) suggested that it might reflect a different blood supply. Hematogenous parenchymal lung metastases reach the lung via the pulmonary arterial circulation, whereas tracheal metastases traverse the bronchial artery, which forms only a small fraction of the systemic circulation.
According to Fournel et al, (6) $95 \%$ of lung metastases are found on CT scans, and only $55 \%$ of endobronchial lesions are identified on CT scans. Airway lesions tend to be overlooked more often than lung parenchyma lesions in patients with colorectal cancer. In this case, endotracheal metastasis manifesting a well enhancing mass was located in the upper trachea, at nearly the subglottic level on the CT scan. It could have been missed easily due to its superior position. Galbis Caravajal et al. (2) reported a patient with endotracheal metastasis from sigmoid colon cancer who had a missed endotracheal metastasis initially on CT scans, which had been found on a routine bronchoscopic exam.

Conti et al reported the tracheal metastasis from colon carcinoma with a stage T4N1M1. 13 months after the initial 
diagnosis, bronchoscopy due to patient's stridor revealed a high-grade tracheal obstruction due to tumor studding. The patient expired 12 months after the diagnosis of endotracheal metastasis (7). Meanwhile, Galbis Caravajal et al. (2) reported two patients who had endotracheal metastases from colon adenocarcinoma. One was diagnosed with sigmoid adenocarcinoma and, 54 months after diagnosis, a pulmonary nodule and single endotracheal lesion were found. The other patient was diagnosed with rectal adenocarcinoma and 14 months after diagnosis pulmonary nodules and two endotracheal lesions were found. The two patients presented the survival duration of 14 and 5 months, respectively, after the diagnosis of endotracheal metastases.

CT has been known to reveal endotracheal nodules, or eccentric tracheal wall thickening with endo- and exophytic tumor growth in the trachea $(2,8,9)$. CT provides valuable information regarding the location of the tumor, type of lung parenchymal lesion, and lymph node status (9). Fournel et al. (6) reported the bronchoscopic findings of a few endobronchial metastases from colorectal cancer. Endoscopically, these lesions appear as bulging or polypoid masses with necrotic components that bleed easily (6). Evaluation using bronchoscopy is limited to the distal part of the obstructing lesion due to luminal narrowing.

Therapeutic options for tracheal metastases include surgery, laser bronchoscopy, radiation therapy, and chemotherapy (7). External and/or internal radiotherapy and laser therapy resection are known to be effective in reducing endotracheal obstruction and controlling hemoptysis (10). Once endotracheal metastasis has developed, the prognosis worsens.

Patients with middle-stage colorectal cancer may present with tracheal metastasis. Such lesions appear as solitary, smooth, polypoid masses in the upper trachea. When an endotracheal nodule is present on postoperative CT scan of patients with malignancies, a primary or secondary endotracheal tumor needs to be distinguished from endotracheal phlegm, which disappears on follow-up CT scan or after vigorous coughing (8). The medical team should evaluate the trachea in patients with colorectal cancer and pulmonary metastases, especially in the later stages of disease. The early detection of subclinical tracheal metastasis during periodic postoperative follow-up examinations would prevent overt tracheal obstruction and improve the life span as well as the quality of life.

Informed Consent: Written informed consent was obtained from patients who participated in this study.

Peer-review: Externally peer-reviewed.

Author contributions: Concept - K.Y.L., J.W.U., J.S.S.; Design - J.Y.C.; Supervision - K.Y.L.; Resource - J.W.U., J.S.S.; Materials - I.Y.C.; Data Collection\&/or Processing - I.Y.C., B.K.J.; Analysis\&/or Interpretation - K.Y.L., J.H.L., S.H.L.; Literature Search - J.Y.C., I.Y.C.; Writing - I.Y.C.; Critical Reviews - K.Y.L.

Conflict of Interest: No conflict of interest was declared by the authors. Financial Disclosure: No financial disclosure was declared by the authors.

\section{References}

1. Froudarakis $M E$, Bouros D, Siafakas NM. Endoluminal metastases of the tracheobronchial tree :is there any way out? Chest 2001;119:679-81. [CrossRef]

2. Galbis Caravajal JM, Sales Badia JG, Trescoli Serrano C, Cordero Rodriguez P, Jorda Aragon C, Naval Sendra E. Endotracheal metastases from colon adenocarcinoma. Clin Transl Oncol 2008;10:676-8. [CrossRef]

3. Hermann A, Schamaun M, Spiegel M. [Endobronchial metastases of extrathoracic malignancies]. Schweiz Med Wochenschr 1982:112:215-7.

4. Shavit L, Maly B, Rosenbaum E, Grenader T. Endotracheal metastases in renal cell carcinoma:A life-threatening but treatable complication. Eur J Intern Med 2007;18:161-3. [CrossRef]

5. Koyi $\mathrm{H}$, Branden $\mathrm{E}$. Intratracheal metastasis from malignant melanoma. J Eur Acad Dermatol Venereol 2000;14:407-8. [CrossRef]

6. Fournel C, Bertoletti L, Nguyen B, Vergnon JM. Endobronchial metastases from colorectal cancers:natural history and role of interventional bronchoscopy. Respiration 2009;77:63-9. [CrossRef]

7. Conti JA, Kemeny N, Klimstra D, Minsky B, Rusch V. Colon carcinoma metastatic to the trachea. Report of a case and a review of the literature. Am J Clin Oncol 1994;17:227-9. [CrossRef]

8. Chong S, Kim TS, Han J. Tracheal metastasis of lung cancer:CT findings in six patients. AJR Am J Roentgenol 2006;186:220-4. [CrossRef]

9. Nair S, Kumar P, Ladas G. Intratracheal metastasis secondary to soft tissue liposarcoma. Singapore Med J 2007;48:e81-3.

10. Katsimbri PP, Bamias AT, Froudarakis ME, Peponis IA, Constantopoulos SH, Pavlidis NA. Endobronchial metastases secondary to solid tumors:report of eight cases and review of the literature. Lung Cancer 2000;28:163-70. [CrossRef] 